\title{
3-S29-1 Symposium
}

\section{Involvement of RAN translation products and neurodegeneration in a triplet repeat disease, FXTAS.}

\section{Norifumi Shioda $^{1}$}

${ }^{\text {I} D e p t . ~ G e n o m i c ~ N e u r o l ., ~ I n s . ~ M o l . ~ E m b r y o l . ~ G e n e t ., ~ K u m a m o t o ~ U n i v . ~}$

In pathological mechanism of repeat diseases, "RNA toxicity" is observed, which repeat-derived RNA binds to various RNA binding proteins, thereby causing abnormalities in RNA metabolism. In addition to its RNA toxicity, "RAN translation" has been found, in which repeat RNA initiates translation in a start codon (AUG) independent manner. In many neurological diseases caused by abnormal elongation of repeat sequences, polypeptides derived from repeat RNA are accumulated in cells. In fragile X-related tremor/ataxia syndrome (FXTAS), which is a triplet repeat disease, elongated CGG repeats in the 5 untranslated region of FMR1 mRNA elicits RAN translation, polyglycine-containing protein (FMRpolyG). In this study, we analyzed the binding protein with FMRpolyG and investigated its involvement with FXTAS pathology. Using immunohistochemistry, FMRpolyG was observed in mouse A9 monochromosomal hybrid cells that harbor whole human X chromosome derived from FXTAS, but not in cells derived from a normal individual. In order to identify binding proteins to FMRpolyG, cell lysates of mouse A9 cells carrying the X chromosome from FXTAS were immunoprecipitated using FMRpolyG antibody, and shotgun proteomics analysis was performed. As a result, it was revealed that causative molecules of some repeat diseases and exosome-related proteins bind to FMRpolyG. These results suggest that we can clarify the mechanisms involved in the onset of other neurological diseases through research on FXTAS. 